\title{
Elastic Registration of Edges Using Diffuse Surfaces
}

\author{
Stefan Fürtinger ${ }^{1}$, Stephen L. Keeling ${ }^{2}$, Gernot Plank ${ }^{3}$ and Anton Prassl ${ }^{4}$
}

\begin{abstract}
In this work, edge sets are mapped one to the other by representing these zero area sets as diffuse images which have positive measure supports that can be registered elastically. The driving application for this work is to map a Purkinje fiber network in the epicardium of one heart to the epicardium of another heart. The approach is to register sufficiently accurate diffuse surface representations of two epicardia and then to apply the resulting transformation to the points of the Purkinje fiber network. To create a diffuse image from a given edge set, a region growing method is used to approximate diffusion of brightness from an edge set to a given point. To be minimized is the sum of squared differences of the transformed diffuse images along with a linear elastic penalty for the registration transformation. A Newton iteration is employed to solve the optimality system, and the degree of diffusion is larger in initial iterations while smaller in later iterations so that a desired local minimum is selected by means of vanishing diffusion. Favorable results are shown for registering highly detailed cardiac geometries.
\end{abstract}

\section{Introduction}

The heart is an electrically controlled mechanical organ whose main function is to pump blood around the circulatory system. Under healthy conditions the heart fulfills this vital objective with remarkable efficiency thanks to a highly organized sequence of events referred to as electro-mechanical coupling. The fast and regular distribution of the electrical impulse which leads to electrical activation of the cardiac tissue is of critical importance in this context. The Purkinje system (PS), that is the specialized conduction system of the main pumping chambers of the heart referred to as ventricles, plays an essential role in this process by conducting electrical excitation wavefronts from the atrio-ventricular node to the endocardium, i.e. the inner surfaces of the ventricular cavities. Due to the high conduction velocities and the network-like topology of the PS the entire endocardium is electrically activated almost simultaneously, thus triggering a highly synchronized mechanical action of the heart.

Histological investigations revealed that the PS consists of a highly ramified network of thin cable-like structures beneath the epithelial layer of the endocardium. The PS is electrically isolated from the ventricular muscle, except at discrete endpoints referred to as Purkinje-Ventricular junctions (PVJs) [27]. Transmission of the electrical signals at these discrete junctional sites is essential to excite the ventricular mass [15], since a loss of electrical synchronicity may entail a severe impairment of cardiac function, which, ultimately, may even lead to sudden cardiac death. The electro-anatomical characteristics of the PS varies significantly between species, but may also vary inter-individually. For instance, the location of PVJs within the ventricular wall is quite distinct between species. In sheep [2] and pig [14] the penetration depth of the PS appears almost fully transmural, whereas in the ventricles of dogs, humans and rabbits [27] PVJs tend to be located rather in sub-endocardial layers.

While recent advances in experimental methodology have allowed more detailed characterizations of cardiac function, quite often further progress is hampered by the inability of current experimental techniques to resolve, with sufficient accuracy, electrical behavior confined to the depth of the ventricles or within the PS. Computer models quite naturally suggest themselves as a surrogate technique to bridge the gap between experimental observations, typically recorded from the surfaces of the heart, and electrical events occurring within the PS or in the depth of ventricular walls. Owing to the physiological importance of the PS it is therefore crucial to account for its role in cardiac function in health and disease, however, despite major recent advancements in modeling technology

\footnotetext{
${ }^{1}$ Institute for Mathematics and Scientific Computing, University of Graz, Austria, email: stefan.fuertinger@uni-graz.at.

${ }^{2}$ Institute for Mathematics and Scientific Computing, University of Graz, Austria, email: stephen.keeling@uni-graz.at.

${ }^{3}$ Institute for Biophysics, Medical University Graz, Austria gernot . plank@medunigraz . at.

${ }^{4}$ Institute for Biophysics, Medical University Graz, Austria anton . prass lemedunigraz . at.

The authors were supported by the Austrian Science Fund Fonds zur Förderung der Wissenschaftlichen Forschung (FWF) under grant SFB F032 and are linked on the SFB webpage http://math. uni-graz.at/mobis/.
} 
[25, 24], the integration of the complex PS topology with anatomically realistic and biophysically detailed models of the ventricles remains a challenge.

The main goal of the presented work is the development of a mathematical framework suitable for mapping the endocardial PS between different ventricular surface geometries. Since detailed experimental characterizations of the network topology of the PS were not available, a reduced model of the PS based on data from literature [29], was constructed and integrated with the San Diego rabbit heart model [28] which served as a template model. For the sake of developing and testing the mapping technique, a recent anatomically realistic model of the rabbit ventricles [4] served as a reference geometry (see models in Fig. 2). We first seek a geometric transformation to match the template heart model to the given reference model. In the context of mathematical image processing this can be seen as 3D registration problem. Once the transformation is found it can be applied to map structures (like the PS) within the template model onto the reference model. This approach guarantees that not only topological features of the PS but also its position relative to the ventricle are preserved and projected onto the reference heart.

Given the sheer size of the models considered here would necessitate a massive computational effort to calculate only simple transformations. Hence we developed a method that is capable of computing even highly non-linear transformations within reasonable time frames while requiring only moderate computational resources. We reduced the dimensionality of the problem by treating the 3D models as sequence of $2 \mathrm{D}$ edges. This strategy reduces memory consumption considerably while simultaneously allowing us to use very efficient techniques to solve the occurring $2 \mathrm{D}$ registration problems.

\section{Edges as Binary Images}

We slice up 3D models and thus obtain two-dimensional cuts. From a mathematical point of view these cuts can be seen as curves. Hence let $\Gamma_{0}$ and $\Gamma_{1}$ denote two curves in $\mathbb{R}^{2}$. Depending on the chosen cutting direction these curves are potentially very non-smooth. However, we may safely assume that both curves are not infinitely long which in mathematical terms means that their Hausdorffmeasure is finite, i.e., $\mathscr{H}^{1}\left(\Gamma_{i}\right)<\infty$ for $i=0,1$. Since we want to approach the problem using image processing techniques we interprete $\Gamma_{0}$ and $\Gamma_{1}$ not as curves but as edges. In doing so let $\Omega:=(0,1)^{2} \subset \mathbb{R}^{2}$ denote our image domain and define $I_{0}$ and $I_{1}$ to be the characteristic functions of $\Gamma_{0}$ and $\Gamma_{1}$ respectively. In this manner $I_{0}$ and $I_{1}$ can be seen as edge maps since $\operatorname{Rg}\left(I_{i}\right)=\{0,1\}$. In other words $I_{0}$ and $I_{1}$ are binary images on $\Omega$. As stated above the objective is to find a transformation to match one heart model to another. Since we do no longer consider 3D models but rather 2D cuts of those models the reformulated objective is now to find a displacement $\mathbf{w}: \mathbb{R}^{2} \rightarrow \mathbb{R}^{2}$ such that $I_{0}(\mathbf{x}+\mathbf{w}(\mathbf{x})) \approx I_{1}(\mathbf{x})$ for all $\mathbf{x} \in \Omega$.

One approach to the computation of the desired displacement is to treat points on $\Gamma_{0}$ as if connected to one another by elastic springs which are perturbed minimally in order to meet the target set $\Gamma_{1}$. However, the computational complexity of such a formulation is very high in relation to the conceptually comparable strategy pursued here. The present strategy is to embed the edges into images which are then registered elastically. Minimizing a linear elastic potential in the registration scheme involving $\Gamma_{0}$ and $\Gamma_{1}$ can be seen as an approximation to the afore mentioned approach. Furthermore, elastic potential energy being used by many authors to regularize image registration is well established and investigated; see, e.g., [23],[16] and particularly the review in [18]. Such regularization is particularly natural when used to register images of tissues having undergone relatively small displacements. However, in the present context, the required displacement field is highly nonlinear, owing partly to the complex geometry of the heart and partly to the great difference in regularity of the two given edge sets. Nevertheless elastic registration is employed here, but with considerable precautions.

Besides choosing an appropriate regularization for the deformation field a suitable notion of similarity of binary images has to be selected as well. Assuming that $\left\{I_{i}\right\}_{i=0,1} \subset L^{2}(\Omega)$, a standard choice (see for instance [9]) is the sum of squared intensity differences (SSID) which in this case is given by

$$
\frac{1}{2} \int_{\Omega}\left|I_{0} \circ(\mathrm{Id}+\mathbf{w})-I_{1}\right|^{2} .
$$

However, in this form the SSID-measure is not feasible for the problem: due to the assumption $\mathscr{H}^{1}\left(\Gamma_{i}\right)<\infty$ both $\Gamma_{0}$ and $\Gamma_{1}$ are sets of Lebesgue-measure zero in $\Omega$ and $\operatorname{supp}\left(I_{i}\right)=\Gamma_{i}$ for $i=0,1$. Hence the trivial deformation $\mathbf{w} \equiv 0$ minimizes the SSID measure (1). A more natural approach to 
measure the difference of edges is employing the Hausdorff-distance:

$$
d_{H}\left(\Gamma_{0}, \Gamma_{1}\right):=\max \left(\sup _{\mathbf{x} \in \Gamma_{0}} d_{\Gamma_{1}}(\mathbf{x}), \sup _{\mathbf{x} \in \Gamma_{1}} d_{\Gamma_{0}}(\mathbf{x})\right),
$$

where

$$
d_{\Gamma_{i}}(\mathbf{x}):=\inf _{\mathbf{y} \in \Gamma_{i}}|\mathbf{x}-\mathbf{y}|_{2}, i=0,1
$$

and $|\cdot|_{2}$ denotes the standard Euclidean norm in $\mathbb{R}^{2}$. In image processing and computer graphics the Hausdorff-distance mainly appears in shape recognition problems. For instance, Knauer et al. [17] developed a method for minimizing the Hausdorff-distance under translations and rigid motions to determine a registration in the context of neurosurgical operations. Though their proposed algorithm is efficient, it is limited to rigid transformations. Fuchs et al. [10] introduced an elastic deformation distance in a shape space; however, calculating the shortest path between shapes proved to be computationally expensive. Droske and Ring [8] developed a regularized shape gradient descent algorithm within a level-set framework for simultaneous registration and segmentation. However, the present problem still lacks sufficient structure to be treated directly by such approaches.

We present here a technique that combines the simplicity of the SSID-measure (1) with the accuracy of the Hausdorff-distance (2). Instead of working with the raw binary edge maps $I_{0}$ and $I_{1}$ we approximate those edge-sets by diffuse regions in images. In other words we use fuzzy edge maps. Note that this approach is not new: Yang et al. [30] employed a similar technique to perform a non-rigid registration of cell nuclei. However, the mathematical idea originates in the famous paper by Ambrosio and Tortorelli [1] who used fuzzy edge-maps (called phase functions) to approximate the Mumford-Shah functional [19]. To obtain fuzzy edges based on the given raw binary images let $\varepsilon>0$ denote a blurring parameter and define $\mathscr{I}_{i}^{\varepsilon}$ for $i=0,1$ by

$$
\mathscr{I}_{i}^{\varepsilon}(\mathbf{x}):= \begin{cases}1-d_{\Gamma_{i}}(\mathbf{x}) / \varepsilon, & \text { if } d_{\Gamma_{i}}(\mathbf{x}) \leq \varepsilon \\ 0, & \text { otherwise }\end{cases}
$$

Then $\mathscr{I}_{i}^{\varepsilon}=1$ where $I_{i}=1$ (i.e., on edges) and smoothly decreases from there. That means the nonzero regions of $\mathscr{I}_{i}^{\varepsilon}$ are diffuse extensions of the edges $\Gamma_{i}$. Note that in practice we do not explicitly calculate the computationally expensive distance function appearing in (3) but rather employ a marching procedure in which the distance $d_{\Gamma_{i}}(\mathbf{x})$ is approximated by successive discrete convolutions. The extent of this "region-growing" depends on the magnitude of $\varepsilon$ : the smaller $\varepsilon$ the less pronounced is the blurring around the edges. In the image processing community this procedure is known as a distance transform (for an overview on the use of distance transforms in image processing see e.g. [26]). Its use in the context of registration problems is also well established: for instance in [13] the authors used constrained distances in the context of interactive non-rigid registration. A variational approach to match distance functions was presented in [22].

Since $\operatorname{supp}\left(\mathscr{I}_{i}^{\varepsilon}\right)$ has positive Lebesgue-measure for any $\varepsilon>0$ for $i=0,1$ the SSID-measure (1) is applicable:

$$
S^{\varepsilon}(\mathbf{w}):=\frac{1}{2} \int_{\Omega}\left|\mathscr{I}_{0}^{\varepsilon} \circ(\mathrm{Id}+\mathbf{w})-\mathscr{I}_{1}^{\varepsilon}\right|^{2} .
$$

Thus we obtain an adapted distance measure for the edges $I_{0}$ and $I_{1}$ which forms the first part of our cost functional. For computing a deformation field $\mathbf{w}$ meeting the present problem's requirements an additional regularization term is needed. As indicated above, we seek elastic deformations. Thus we employ the following linear elastic potential (compare for instance [18])

$$
E(\mathbf{w}):=\frac{\lambda}{2} \int_{\Omega}(\nabla \cdot \mathbf{w})^{2} d \mathbf{x}+\frac{\mu}{4} \int_{\Omega}\left|\nabla \mathbf{w}^{\top}+\nabla \mathbf{w}\right|_{F}^{2},
$$

where $|\cdot|_{F}$ denotes the Frobenius-norm and $\mu$ and $\lambda$ are positive constants describing the elastic properties of the body, the so-called Navier-Lamé constants. Thus we obtain the following cost functional

$$
\begin{aligned}
J^{\varepsilon}(\mathbf{w}) & :=S^{\varepsilon}(\mathbf{w})+E(\mathbf{w}) \\
& =\frac{1}{2}\left\|\mathscr{I}_{0}^{\varepsilon} \circ(\mathrm{Id}+\mathbf{w})-\mathscr{I}_{1}^{\varepsilon}\right\|_{L^{2}(\Omega)}^{2}+\frac{\lambda}{2}\|\nabla \cdot \mathbf{w}\|_{L^{2}(\Omega)}^{2}+\frac{\mu}{4}\left\|\nabla \mathbf{w}^{\top}+\nabla \mathbf{w}\right\|_{L^{2}(\Omega)}^{2},
\end{aligned}
$$


for a fixed $\varepsilon>0$. The cost $J$ is well defined for $\mathbf{w} \in H^{1}(\Omega)$. Thus we want to solve the minimization problem

$$
\min _{\mathbf{w} \in H^{1}(\Omega)} J^{\varepsilon}(\mathbf{w}) .
$$

Here several important observations should be made. The fuzzy edges $I_{i}^{\varepsilon}$ vary with the value of $\varepsilon$. Hence for each $\varepsilon$, (6) forms a stand-alone minimization problem. This immediately gives rise to the following questions. For fixed $\varepsilon>0$, does (6) have a solution, i.e., is the use of min instead of inf justified? Assuming that the first question can be answered positively, what are the asymptotic properties of these solutions as $\varepsilon \rightarrow 0$ ? We devote ourselves to both questions in the following subsection.

\subsection{Properties of $J^{\varepsilon}$}

Due to the lack of space the first question concerning the existence of solutions to (6) for a given $\varepsilon>0$ cannot be answered in full detail. However, in the following we will outline the basic idea of the proof.

Below, we use the notation $\mathbf{A}: \mathbf{B}=\sum_{i j} A_{i j} B_{i j}$ for matrices $\mathbf{A}=\left\{A_{i j}\right\}$ and $\mathbf{B}=\left\{B_{i j}\right\}$. We introduce the space of infinitesimal rigid motions

$$
\mathrm{RM}=\left\{\mathbf{w}=\mathbf{c}+\mathbf{W x}: \mathbf{c} \in \mathbb{R}^{d}, \mathbf{W} \in \mathbb{S}^{d}\right\}
$$

where

$$
\mathbb{S}^{d}=\left\{\mathbf{W} \in \mathbb{R}^{d \times d}: \mathbf{W}+\mathbf{W}^{\mathrm{T}}=0\right\} .
$$

Furthermore, we define the following closed linear subspace of $H^{1}(\Omega)$ :

$$
\mathscr{H}=\left\{\mathbf{w} \in H^{1}(\Omega): \int_{\Omega} \mathbf{w}(\mathbf{x}) d \mathbf{x}=0, \int_{\Omega}\left[\mathbf{w}(\mathbf{x}) \mathbf{x}^{\mathrm{T}}-\mathbf{x w}(\mathbf{x})^{\mathrm{T}}\right] d \mathbf{x}=0\right\} .
$$

Straight forward calculations show that $J^{\varepsilon}$ is bounded on $H^{1}(\Omega)$. However, it is easy to see that the whole of RM is in the kernel of the elastic penalty $E$ in (5), i.e., $\mathrm{RM} \subset \operatorname{ker}(E)$. Thus

$$
c\|\mathbf{u}\|_{H^{1}(\Omega)}^{2} \not J^{\varepsilon}(\mathbf{u})=S^{\mathcal{E}}(\mathbf{u}) \leq 2|\Omega|, \quad \forall \mathbf{u} \in \mathrm{RM},
$$

which means that $J^{\varepsilon}$ is not coercive on $H^{1}(\Omega)$. However, it can be shown that the cost $J^{\varepsilon}$ is coercive and bounded on $\mathscr{H}$. Therefore we established the following direct sum decomposition of $H^{1}(\Omega)$.

Theorem $1 H^{1}(\Omega)=\mathrm{RM} \oplus \mathscr{H}$.

Proof 1 A rigorous proof is given in [11].

This decomposition is key to proving existence of a minimizing element of (6) in $H^{1}(\Omega)$. Under further assumptions existence of (in a certain sense) minimizing rigid motions can be shown. Conversely, given $\mathbf{u} \in \mathrm{RM}$ there exists a $\mathbf{v}^{*} \in \mathscr{H}$ such that

$$
J^{\varepsilon}\left(\mathbf{u}+\mathbf{v}^{*}\right)=\inf _{\mathbf{v} \in \mathscr{H}} J^{\varepsilon}(\mathbf{u}+\mathbf{v}) .
$$

For the proof we also refer to [11]. Following Theorem 1 and using these two results existence of a minimizer of $J^{\varepsilon}$ for $\varepsilon>0$ fixed is guaranteed by the following

Theorem 2 Let $\varepsilon>0$ be fixed, $\mathscr{I}_{0}^{\varepsilon} \in W^{1, \infty}(\Omega), \mathscr{I}_{1}^{\varepsilon} \in L^{\infty}(\Omega)$ and $\lambda, \mu>0$. Then there exists a $\mathbf{w}^{\star} \in H^{1}(\Omega)$ such that

$$
J^{\varepsilon}\left(\mathbf{w}^{\star}\right)=\min _{w \in H^{1}(\Omega)} J^{\varepsilon}(\mathbf{w}) .
$$

Proof 2 A rigorous proof is given in [11].

Note that by definition (3) both fuzzy edges $\mathscr{I}_{i}^{\varepsilon}$ satisfy the regularity assumptions stated in Theorem 2, provided the the edge sets $\Gamma_{i}$ are sufficiently regular. Under such assumptions, (6) is a well posed minimization problem for each $\varepsilon>0$.

Having guaranteed existence of minimizers for each fixed $\varepsilon$ we may now address the question of asymptotic behavior of solutions to (6) as $\varepsilon \rightarrow 0$. Looking at definition (3) it is easy to see that the fuzzy edges $\mathscr{I}_{i}^{\varepsilon}$ converge pointwise to the binary images $I_{i}$ as $\varepsilon \rightarrow 0$. However, as pointed out above for $\varepsilon=0$ the trivial deformation $\mathbf{w}=0$ minimizes the similarity measure (4). Indeed, we could show the following convergence of minimizers as $\varepsilon \rightarrow 0$. 
Theorem 3 Assume the conditions of Theorem 2. For every $\varepsilon>0$, let $\mathbf{w}_{\varepsilon} \in H^{1}(\Omega)$ denote the minimizer of $J^{\varepsilon}(\mathbf{w})$. Let $\mathbf{u}_{\varepsilon}$ and $\mathbf{v}_{\varepsilon}$ denote the projections of $\mathbf{w}_{\varepsilon}$ onto RM and $\mathscr{H}$, respectively. Then

$$
\lim _{\varepsilon \rightarrow 0} \mathbf{v}_{\varepsilon}=0
$$

Also, there exists a $\mathbf{u}_{0} \in R M$ such that

$$
\lim _{\mathcal{\varepsilon} \rightarrow 0} S^{\mathcal{\varepsilon}}\left(\mathbf{u}_{0}+\mathbf{v}_{\mathcal{\varepsilon}}\right)=\lim _{\varepsilon \rightarrow 0} S^{\varepsilon}\left(\mathbf{u}_{\varepsilon}+\mathbf{v}_{\varepsilon}\right)=0 .
$$

Proof 3 A rigorous proof is given in [11].

For a better understanding of the behavior of the minimization problem (6) as $\varepsilon \rightarrow 0$ we present a simplified 1D-example.

Remark 1 Let $\Omega:=(0,1) \subset \mathbb{R}$ and consider the "binary images" $I_{0}$ and $I_{1}$ defined by

$$
I_{0}(x):=\left\{\begin{array}{ll}
1, & x=\frac{1}{4}, \\
0, & \text { otherwise, }
\end{array} \quad I_{1}(x):= \begin{cases}1, & x=\frac{1}{2}, \\
0, & \text { otherwise } .\end{cases}\right.
$$

The objective is to find a deformation $w$ such that $I_{0}(x+w(x)) \approx I_{1}(x)$. In analogy to the approach presented above we start with the cost functional

$$
\begin{aligned}
J(w) & :=\int_{0}^{1}\left|I_{0}(x+w(x))-I_{1}(x)\right|^{2} d x+\mu \int_{0}^{1}\left|w^{\prime}(x)\right|^{2} d x \\
& =\mathscr{S}\left(I_{0}, I_{1} ; w\right)+\mathscr{P}(w),
\end{aligned}
$$

with a regularization parameter $\mu>0$. Similar to the $2 D$ case described above $\mathscr{S}\left(I_{0}, I_{1} ; 0\right)=0$ since supp $\left(I_{i}\right)$ consists only of discrete points. Employing the same strategy as in $2 D$ we proceed to fuzzy "edges" by defining

$$
\mathscr{I}_{0}^{\varepsilon}(x):=\left\{\begin{array}{ll}
1, & \frac{1}{4} \leq x \leq \frac{1}{4}+\varepsilon, \\
0, & \text { otherwise },
\end{array} \quad \mathscr{I}_{1}^{\varepsilon}(x):=\left\{\begin{array}{ll}
1, & \frac{1}{2} \leq x \leq \frac{1}{2}+2 \varepsilon, \\
0, & \text { otherwise },
\end{array} \quad 0<\varepsilon \leq \frac{1}{4},\right.\right.
$$

and thus modify the cost accordingly:

$$
J^{\varepsilon}(w):=\mathscr{S}\left(\mathscr{I}_{0}^{\varepsilon}, \mathscr{I}_{1}^{\varepsilon} ; w\right)+\mathscr{P}(w) .
$$

Since $\mathscr{I}_{i}^{\varepsilon}$ are step functions (see Fig. $1(a)$ ) we obviously look for deformations of the form $w_{\varepsilon}(x):=$ bx with some non-negative scalar $b$ and a fixed $0<\varepsilon \leq \frac{1}{4}$. In this very simple case it is possible to explicitly write down what the desired deformation should be in order to get a perfect registration: $w_{\varepsilon}^{\star}(x):=x$, thus $b^{\star}=1$. In the following we investigate the properties of the modified cost $J^{\varepsilon}$ given the desired solution $w_{\varepsilon}^{\star}(x)=x$ versus the trivial deformation $w=0$.

First note that the penalty $\mathscr{P}$ for deformations of the form $w_{\varepsilon}=b x$ is given by

$$
\mathscr{P}\left(w_{\varepsilon}\right)=\mu \int_{0}^{1}|b|^{2} d x=\mu b^{2}
$$

thus

$$
\mathscr{P}\left(w_{\varepsilon}^{\star}\right)=\mu, \quad \text { and } \quad \mathscr{P}(0)=0 .
$$

Furthermore, the desired solution minimizes the (adapted) similarity measure

$$
\mathscr{S}\left(\mathscr{I}_{0}^{\varepsilon}, \mathscr{I}_{1}^{\varepsilon} ; w_{\varepsilon}^{\star}\right)=0
$$

On the other hand for the trivial deformation we compute

$$
\mathscr{S}\left(\mathscr{I}_{0}^{\varepsilon}, \mathscr{I}_{1}^{\varepsilon} ; 0\right)=\left(\left(\frac{1}{4}+\varepsilon\right)-\frac{1}{4}\right)+\left(\frac{1}{2}+2 \varepsilon-\frac{1}{2}\right)=3 \varepsilon .
$$

Now choose $0<\varepsilon<\min \left(\frac{1}{4}, \frac{\mu}{3}\right)$. Then relations (10), (11) and (12) imply

$$
\begin{aligned}
J^{\varepsilon}(0)=\mathscr{S}\left(\mathscr{I}_{0}^{\varepsilon}, \mathscr{I}_{1}^{\varepsilon} ; 0\right)+\mathscr{P}(0) & =3 \varepsilon+0 \\
& <0+3 \cdot \frac{\mu}{3} \\
& =\mathscr{S}\left(\mathscr{I}_{0}^{\varepsilon}, \mathscr{I}_{1}^{\varepsilon} ; w_{\varepsilon}^{\star}\right)+\mathscr{P}\left(w_{\varepsilon}^{\star}\right)=J^{\varepsilon}\left(w_{\varepsilon}^{\star}\right) .
\end{aligned}
$$




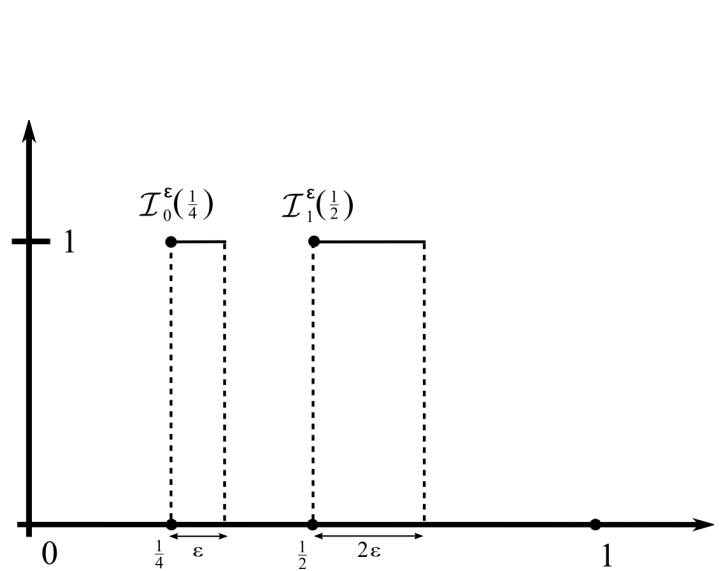

(a)

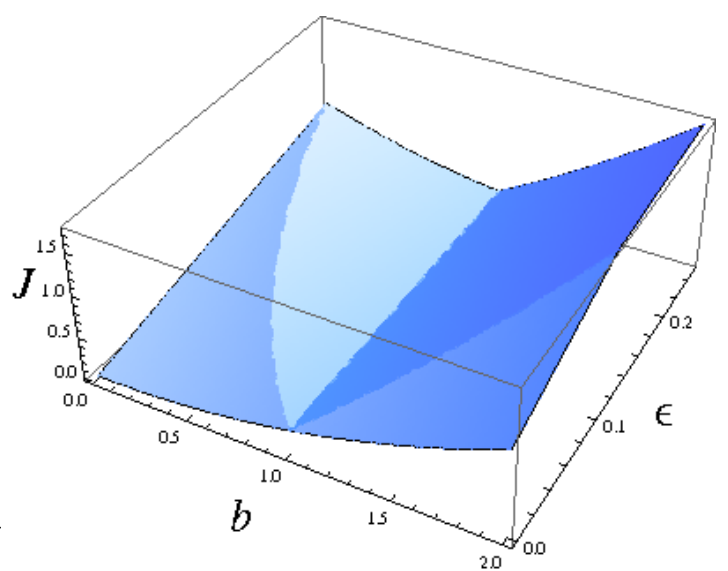

(b)

Figure 1: (a) Sketch of the simplified 1D-example discussed in Remark 1. (b) Graph of $G(\varepsilon, b)$ on $\left[0, \frac{1}{4}\right] \times[0,2]$ for $\mu=\frac{1}{4}$.

The crucial observation to make here is that although $\varepsilon>0$ the trivial deformation minimizes the $\operatorname{cost} J^{\varepsilon}$. A closer look at the calculations shows that there is an important relation between the constant $\mu$ and $\varepsilon$ : in the above example $\varepsilon$ was chosen "too small" compared to the regularization parameter $\mu$ so that the similarity measure was minimized by $w=0$ instead of the desired deformation. To illustrate this phenomenon Fig. 1 (b) shows the graph of $G(\varepsilon, b):=J^{\varepsilon}(b x)$ for $\varepsilon \in\left[0, \frac{1}{4}\right]$, $b \in[0,2]$ and $\mu=\frac{1}{4}$. It can be seen that for small values of $\varepsilon$ the global minimizer of $J^{\varepsilon}$ is $b=0$ (and hence $w=0$ ) whereas for even very large values of $\varepsilon$ the cost $J^{\varepsilon}$ is (globally) minimized by $b=1$, i.e., the desired deformation.

It will be demonstrated in a separate work, using techniques of De Giorgi [6, 3], that an $\varepsilon$ dependent normalization of (4) $\Gamma$-converges as $\varepsilon \rightarrow 0$ to (2), for which the global minimizer is not $\mathbf{w}=0$. Nevertheless, the $1 D$ example given above demonstrates that it is simpler to seek local minima of the functional $J^{\varepsilon}$ presented here. Note that the behavior manifested in the $1 D$ example can be seen in $2 D$ as well. For vanishingly small values of $\varepsilon$ (small compared to the Navier-Lamé constants) $J^{\varepsilon}$ is minimized by $\mathbf{w}=0$. On the other hand, local minima provide a better registration of the edge sets. This knowledge has led us to the development of an iterative solution strategy that allows to compute first global minima for $\varepsilon$ large, which become desired local minima as $\varepsilon$ gets smaller. Starting with sufficiently diffuse images, we use the computed minimizer for larger values of $\varepsilon$ as an initial guess to solve the registration problem for smaller values of $\varepsilon$. By doing so the computed transformations are refined in the course of the iteration but simultaneously do not get close to zero as $\varepsilon \rightarrow 0$ (details are given in Sect. 3.1). This strategy proved to be remarkably robust in practice and produced very promising results.

\subsection{Optimality Conditions}

We start by deducing the necessary optimality conditions for the minimization problem (6). The Gâteaux derivative of $J^{\mathcal{E}}$ in an arbitrary direction $\mathbf{v} \in C^{\infty}(\bar{\Omega})$ is defined by

$$
\frac{\delta J^{\varepsilon}}{\delta \mathbf{w}}(\mathbf{w} ; \mathbf{v}):=\left.\frac{d}{d s} J^{\varepsilon}(\mathbf{w}+s \mathbf{v})\right|_{s=0} .
$$

For the sake of clarity we compute the derivatives of $S^{\varepsilon}$ and $E$ separately. Starting with $S^{\varepsilon}$ we get

$$
\frac{\delta S^{\varepsilon}}{\delta \mathbf{w}}(\mathbf{w} ; \mathbf{v})=\int_{\Omega}\left(\mathscr{I}_{0}^{\varepsilon} \circ(\mathrm{Id}+\mathbf{w})-\mathscr{I}_{1}^{\varepsilon}\right) \mathbf{v} \cdot \nabla \mathscr{I}_{0}^{\varepsilon} \circ(\mathrm{Id}+\mathbf{w}),
$$

and for $E(\mathbf{w})$ we obtain

$$
\begin{aligned}
\frac{\delta E}{\delta \mathbf{w}}(\mathbf{w} ; \mathbf{v})= & \int_{\Omega} \mu\left(\nabla \mathbf{w}^{\top}+\nabla \mathbf{w}\right):\left(\nabla \mathbf{v}^{\top}+\nabla \mathbf{v}\right) d \mathbf{x} \\
& +\int_{\Omega} \lambda(\nabla \cdot \mathbf{w})(\nabla \cdot \mathbf{v}) .
\end{aligned}
$$


Assuming sufficient regularity of $\mathbf{w}$ we will deduce a strong optimality formulation for (6). Therefore we first use partial integration in (14)

$$
\begin{aligned}
\frac{\delta E}{\delta \mathbf{w}}(\mathbf{w} ; \mathbf{v})= & \mu \int_{\partial \Omega} \sum_{\ell=1}^{2} v_{\ell}\left(\nabla w_{\ell}+\partial_{x_{\ell}} \mathbf{w}\right) \cdot \mathbf{n}-\mu \int_{\Omega}(\Delta \mathbf{w}+\nabla(\nabla \cdot \mathbf{w})) \cdot \mathbf{v} \\
& +\lambda \int_{\partial \Omega}(\nabla \cdot \mathbf{w})(\mathbf{v} \cdot \mathbf{n})-\lambda \int_{\Omega} \mathbf{v} \cdot \nabla(\nabla \cdot \mathbf{w}),
\end{aligned}
$$

where $\mathbf{n}$ denotes the outer unit normal vector on $\partial \Omega$. The (weak) necessary optimality condition associated to (6) is given by

$$
\frac{\delta J}{\delta \mathbf{w}}(\mathbf{w} ; \mathbf{v})=\frac{\delta S^{\varepsilon}}{\delta \mathbf{w}}(\mathbf{w} ; \mathbf{v})+\frac{\delta E}{\delta \mathbf{w}}(\mathbf{w} ; \mathbf{v})=0, \quad \forall \mathbf{v} \in C^{\infty}(\bar{\Omega}) .
$$

Since (17) holds for any variation $\mathbf{v} \in C^{\infty}(\bar{\Omega})$ we may apply the fundamental Lemma of calculus of variations. Thus using (13) and (16) we obtain the Euler-Lagrange equations, i.e., the strong optimality formulation, associated to the minimization problem (6)

$$
\left\{\begin{aligned}
\mathscr{E} \mathbf{w}-\mathbf{f}\left(\mathbf{x}, \mathbf{w} ; \mathscr{I}_{0}^{\varepsilon}, \mathscr{I}_{1}^{\varepsilon}\right)=0, & \text { in } \Omega, \\
\lambda n_{\ell} \nabla \cdot \mathbf{w}+\mu\left(\nabla w_{\ell}+\partial_{x_{\ell}} \mathbf{w}\right) \cdot \mathbf{n}=0, & \text { on } \partial \Omega,
\end{aligned}\right.
$$

where $\mathscr{E}$ is the elasticity operator defined by

$$
\mathscr{E} \mathbf{w}:=\mu \Delta \mathbf{w}+(\mu+\lambda) \nabla(\nabla \cdot \mathbf{w}),
$$

and

$$
\mathbf{f}\left(\mathbf{x}, \mathbf{w} ; \mathscr{I}_{0}^{\varepsilon}, \mathscr{I}_{1}^{\varepsilon}\right):=\left(\mathscr{I}_{0}^{\varepsilon} \circ(\mathrm{Id}+\mathbf{w})-\mathscr{I}_{1}^{\varepsilon}\right) \nabla \mathscr{I}_{0}^{\varepsilon} \circ(\mathrm{Id}+\mathbf{w}),
$$

is the driving force of the registration. Here several important observations should be made. First note that the strength of the driving force $\mathbf{f}$ determines the magnitude of a deformation field $\mathbf{w}$ that satisfies (18). Furthermore, $\mathbf{f}$ is the Gâteaux derivative of the adapted SSID distance measure (4). Thus if we use a very small $\varepsilon$ (or the raw binary edge maps $I_{i}$ instead of the fuzzy edges $\mathscr{I}_{i}^{\varepsilon}$ in the SSID-distance) the resulting driving force of the registration is zero, a.e., and hence the trivial deformation solves the Euler-Lagrange equations (18) (compare the 1D-example given in Remark 1).

On the other hand large values of $\varepsilon$ indeed generate a sufficiently big driving force in order to prevent the trivial deformation from solving (18). However, choosing $\varepsilon$ too large leads to blurred fuzzy edges $\mathscr{I}_{i}^{\varepsilon}$ and thus a loss of potentially important features of the original edges $\Gamma_{i}$.

The vanishing diffusion strategy proposed here (see Algorithm 1) is designed to address both problems. Starting with $\varepsilon$ large the driving force in (18) provokes a non trivial initial solution $\mathbf{w}$. By iteratively refining the initial deformation field $\mathbf{w}$ we are able to preserve characteristic features of the original edges $\Gamma_{i}$. Thus our solution strategy is robust against the initial choice of (a possibly large) $\varepsilon>0$ while simultaneously allowing us to compute only the desired local minima of $J^{\varepsilon}$ as $\varepsilon$ gets smaller.

\subsection{Solution Strategy}

The strong optimality conditions (18) are a system of nonlinear partial differential equations (PDEs) in w. Thus we linearize (18) by employing Newton's method on the functional $J$ which takes the form

$$
\left\{\begin{aligned}
\frac{\delta^{2} J}{\delta \mathbf{w}^{2}}\left(\mathbf{w}_{k} ; \mathbf{v}, \delta \mathbf{w}_{k}\right) & =-\frac{\delta J}{\delta \mathbf{w}}\left(\mathbf{w}_{k} ; \mathbf{v}\right), \quad \forall \mathbf{v} \in C^{\infty}(\bar{\Omega}), \\
\mathbf{w}_{k+1} & =\mathbf{w}_{k}+\tau \delta \mathbf{w}_{k},
\end{aligned}\right.
$$

where $\tau>0$ denotes a given step-size and $k=1,2, \ldots$ is the iteration index. The Lax-Milgram Lemma [5] may be used to show that (21) admits an unique solution for each fixed $k$.

Theorem 4 Let $\mathbf{v} \in C^{\infty}(\bar{\Omega}), \mathscr{I}_{0}^{\varepsilon} \in W^{1, \infty}(\Omega), \mathscr{I}_{1}^{\varepsilon} \in L^{\infty}(\Omega)$ and $\lambda, \mu>0$. If for given $\mathbf{w} \in H^{1}(\Omega)$

$$
\int_{\Omega}\left|(\mathbf{a}+M \mathbf{x}) \cdot \nabla \mathscr{I}_{0}^{\varepsilon} \circ(I d+\mathbf{w})\right|^{2} d \mathbf{x}=0 \quad \text { implies } \quad \mathbf{a}+M \mathbf{x}=0,
$$

for every skew-symmetric matrix $\mathbf{M} \in \mathbb{R}^{N \times N}$ and every vector $\mathbf{a} \in \mathbb{R}^{N}$ then there exists a unique $\mathbf{u} \in H^{1}(\Omega)$ satisfying $(21)$. 
Proof 4 A rigorous proof is given in [11].

Assumption (22) essentially says that the image $\mathscr{I}_{0}^{\varepsilon} \circ\left(\mathrm{Id}+\mathbf{w}_{k}\right)$ manifests sufficiently few symmetries. Convergence of Newton's method (21) as $k \rightarrow \infty$ for a fixed $\varepsilon>0$ and a suitable initial guess can be shown using the Newton-Kantorovich Theorem (see e.g. [21]).

Similar to the strategy presented in the previous section we will now deduce a strong formulation of (21). For the sake of clarity we compute again the derivatives of $S^{\varepsilon}$ and $E$ separately. Starting with $S^{\varepsilon}$ we get

$$
\frac{\delta^{2} S^{\varepsilon}}{\delta \mathbf{w}^{2}}\left(\mathbf{w}_{k} ; \mathbf{v}, \delta \mathbf{w}_{k}\right)=\int_{\Omega} \mathbf{v}\left[\nabla \mathscr{I}_{0}^{\varepsilon} \circ\left(\mathrm{Id}+\mathbf{w}_{k}\right)\right]\left[\nabla \mathscr{I}_{0}^{\varepsilon} \circ\left(\mathrm{Id}+\mathbf{w}_{k}\right)\right]^{\top} \delta \mathbf{w}_{k} .
$$

By using the first variational derivative (14) of the linear elastic potential we obtain further

$$
\begin{aligned}
\frac{\delta^{2} E}{\delta \mathbf{w}^{2}}\left(\mathbf{w}_{k} ; \mathbf{v}, \delta \mathbf{w}_{k}\right)= & \int_{\Omega} \mu\left(\nabla \mathbf{v}^{\top}+\nabla \mathbf{v}\right):\left(\nabla \delta \mathbf{w}_{k}^{\top}+\nabla \delta \mathbf{w}_{k}\right) \\
& +\int_{\Omega} \lambda(\nabla \cdot \mathbf{v})\left(\nabla \cdot \delta \mathbf{w}_{k}\right) d \mathbf{x},
\end{aligned}
$$

Under stronger regularity assumptions on $\mathbf{w}$ we may again use partial integration and apply the fundamental Lemma of calculus of variations to obtain the strong formulation of (21)

$$
\left\{\begin{array}{cc}
\left(-\mathscr{E}+\left[\nabla \mathscr{I}_{0}^{\varepsilon} \circ\left(\mathrm{Id}+\mathbf{w}_{k}\right)\right]\left[\nabla \mathscr{I}_{0}^{\varepsilon} \circ\left(\mathrm{Id}+\mathbf{w}_{k}\right)\right]^{\top}\right) \delta \mathbf{w}_{k}=\mathscr{E} \mathbf{w}_{k}-\mathbf{f}\left(\mathbf{x}, \mathbf{w}_{k} ; \mathscr{I}_{0}^{\varepsilon}, \mathscr{I}_{1}^{\varepsilon}\right), & \Omega, \\
\lambda n_{\ell} \nabla \cdot \mathbf{w}_{k}+\mu\left(\nabla w_{k_{\ell}}+\partial_{x_{\ell}} \mathbf{w}_{k}\right) \cdot \mathbf{n}=0, & \partial \Omega .
\end{array}\right.
$$

which is the desired linear PDE-system for the unknown function $\delta \mathbf{w}_{k}$.

\section{Numerical Approximation}

We will first introduce a discretization scheme for the strong formulation (23) of the Newton step and then explain the discrete realization of Newton's method (21). Let from now on $\mathbf{w}(\mathbf{x}):=$ $(u(\mathbf{x}), v(\mathbf{x}))^{\top}$, and $\mathbf{x}=(x, y) \in \Omega$. Then we may rewrite the elasticity operator $\mathscr{E}$

$$
\begin{aligned}
\mathscr{E} \mathbf{w} & =\mu\left(\frac{\partial^{2} \mathbf{w}}{\partial x^{2}}+\frac{\partial^{2} \mathbf{w}}{\partial y^{2}}\right)+(\mu+\lambda) \nabla\left(\frac{\partial u}{\partial x}+\frac{\partial v}{\partial y}\right) \\
& =\left(\begin{array}{cc}
(\lambda+2 \mu) \frac{\partial^{2}}{\partial x^{2}}+\mu \frac{\partial^{2}}{\partial y^{2}} & (\lambda+\mu) \frac{\partial^{2}}{\partial x \partial y} \\
(\lambda+\mu) \frac{\partial^{2}}{\partial x \partial y} & \mu \frac{\partial^{2}}{\partial x^{2}}+(\lambda+2 \mu) \frac{\partial^{2}}{\partial y^{2}}
\end{array}\right)\left(\begin{array}{c}
u \\
v
\end{array}\right)=:\left(\begin{array}{ll}
\mathscr{E}_{11} & \mathscr{E}_{12} \\
\mathscr{E}_{21} & \mathscr{E}_{22}
\end{array}\right)\left(\begin{array}{c}
u \\
v
\end{array}\right) .
\end{aligned}
$$

For the sake of simplicity we drop the iteration index $k$ here. Since we are working with digital images we define a grid $\Omega_{h}:=\{1, \ldots, N\}^{2}$, where $N$ denotes the resolution of the images. We use a unit step size $h:=1$, i.e., the width of a cell is one, and employ standard central finite differences to discretize the Newton step (23). In particular let $\mathbf{j}:=\left(j_{1}, \ldots, j_{N}\right) \in \mathbb{R}^{N}$ be an integer component multi index, $\mathbf{1}:=(1, \ldots, 1)^{\top} \in \mathbb{R}^{N}$ and the cell centroids be given by $\mathbf{x}_{j}:=\mathbf{j}, \mathbf{1} \leq \mathbf{j} \leq N \cdot \mathbf{1}$. We denote the array arising from evaluating e.g. $u$ at each grid point by $u\left(\Omega_{h}\right) \in \mathbb{R}^{N \times N}$. Hence $U_{\mathbf{j}} \approx u\left(\mathbf{x}_{j}\right)$ and $\vec{u} \in \mathbb{R}^{N^{2}}$ denotes the vector of values $\left\{U_{\mathbf{j}}\right\}$ corresponding to the lexicographic ordering in which $j_{1}$ increments first from 1 to $N$, then $j_{2}$ and so on. Further, let $\mathbf{D}(\vec{u}) \in \mathbb{R}^{N^{2} \times N^{2}}$ be the diagonal matrix arising from situating the values $\left\{U_{\mathbf{j}}\right\}$ along the diagonal according to lexicographic ordering.

Due to the lack of space we cannot give the discretization of the elasticity operator $\mathscr{E}$ in full detail. Thus we show, for instance, the discretization of $\mathscr{E}_{11}$ near the lower left corner of $\Omega_{h}$

$$
\left(\begin{array}{ccc}
0 & 0 & -2 \mu \\
0 & 8 \mu+4 \lambda & -4 \mu-4 \lambda \\
0 & 0 & -2 \mu \\
0 & 0 & -2 \mu \\
0 & 2 \lambda+4 \mu & -2 \mu-2 \lambda \\
0 & 0 & 0
\end{array}\right) \quad\left(\begin{array}{ccc}
-2 \mu & 0 & -2 \mu \\
-4 \mu-4 \lambda & 16 \mu+8 \lambda & -4 \mu-4 \lambda \\
-2 \mu & 0 & -2 \mu \\
-2 \mu & 0 & -2 \mu \\
-2 \mu-2 \lambda & 8 \mu+4 \lambda & -2 \mu-2 \lambda \\
0 & 0 & 0
\end{array}\right) \cdots
$$


The upper right block represents the stencil weights for neighbors of a field cell. Similarly the other blocks show for boundary cells the stencil weights for their neighbors. With the same format we represent the stencils of $\mathscr{E}_{12}$ :

$$
\begin{gathered}
\left(\begin{array}{ccc}
0 & \mu-\lambda & -\mu-\lambda \\
0 & 0 & 0 \\
0 & -\mu-\lambda & \mu+\lambda
\end{array}\right) \quad\left(\begin{array}{ccc}
\mu+\lambda & 0 & -\mu-\lambda \\
0 & 0 & 0 \\
-\mu-\lambda & 0 & \mu+\lambda
\end{array}\right) \\
\left(\begin{array}{ccc}
0 & \mu-\lambda & -\mu-\lambda \\
0 & \mu+\lambda & -\mu+\lambda \\
0 & 0 & 0
\end{array}\right) \quad\left(\begin{array}{ccc}
\mu+\lambda & 0 & -\mu-\lambda \\
\mu-\lambda & 0 & -\mu-\lambda \\
0 & 0 & 0
\end{array}\right)
\end{gathered}
$$

The stencils for $\mathscr{E}_{22}$ and $\mathscr{E}_{21}$ are constructed by adequate copying and mirroring of (24) and (25) respectively. This gives rise to matrices $\mathbf{E}_{k . \ell} \in \mathbb{R}^{N^{2} \times N^{2}}$ with $1 \leq k, \ell \leq 2$ which form the discrete version of the operator $\mathscr{E}$ under lexicographic ordering, i.e.,

$$
\mathscr{E}\left(u\left(\Omega_{h}\right), v\left(\Omega_{h}\right)\right) \approx\left(\begin{array}{c}
E_{11} \vec{u}+E_{12} \vec{v} \\
E_{21} \vec{u}+E_{22} \vec{v}
\end{array}\right),
$$

so that we may write

$$
\mathbf{E} \vec{w}:=\left(\begin{array}{ll}
\mathbf{E}_{11} & \mathbf{E}_{12} \\
\mathbf{E}_{21} & \mathbf{E}_{22}
\end{array}\right)\left(\begin{array}{c}
\vec{u} \\
\vec{v}
\end{array}\right) .
$$

The discrete version $\tilde{\mathscr{I}}_{1} \in \mathbb{R}^{N \times N}$ of $\mathscr{I}_{1}^{\varepsilon}$ is readily established by setting $\tilde{\mathscr{I}}_{1, \mathbf{j}}:=\mathscr{I}_{1}^{\varepsilon}\left(\mathbf{x}_{j}\right)$. For approximating $\mathscr{I}_{0}^{\varepsilon}(\mathbf{x}+\mathbf{w})$ and $\nabla \mathscr{I}_{0}^{\varepsilon} \circ(\mathrm{Id}+\mathbf{w})$ we use a bilinear interpolation scheme whenever $\mathscr{I}_{0}^{\varepsilon}$ is evaluated at non-grid points and assign the extrapolation value zero if $\mathbf{x}+\mathbf{w}(\mathbf{x})$ lies outside of $\Omega$. Thus we obtain a matrix $\tilde{\mathscr{I}}_{0} \in \mathbb{R}^{N \times N}$. Let $\mathbf{G}\left(\tilde{\mathscr{I}}_{0}\right) \in \mathbb{R}^{2 N^{2} \times 2 N^{2}}$ denote the approximation to $\left[\nabla \mathscr{I}_{0}^{\varepsilon} \circ(\mathrm{Id}+\mathbf{w})\right]\left[\nabla \mathscr{I}_{0}^{\varepsilon} \circ(\mathrm{Id}+\mathbf{w})\right]^{\top}$ and define $\vec{f} \in \mathbb{R}^{2 N^{2}}$ to be the discrete version of the force field $\mathbf{f}\left(\mathbf{x}, \mathbf{w}_{k} ; \mathscr{I}_{0}^{\varepsilon}, \mathscr{I}_{1}^{\varepsilon}\right)$ (further details are given in [12] and [11]). Then the Newton step (23) is discretized by

$$
\left(-\mathbf{E}+\mathbf{G}\left(\tilde{\mathscr{I}}_{0}\right)\right) \delta \vec{w}=-\mathbf{E} \vec{w}-\vec{f}
$$

which is a linear equation system in the unknown $\delta \vec{w} \in \mathbb{R}^{2 N^{2}}$.

\subsection{The Discrete Newton Iteration}

We use $\vec{w}_{1}:=0 \in \mathbb{R}^{2 N^{2}}$ as initial guess and employ the discrete Newton step (26) to obtain the following iteration

$$
\left\{\begin{array}{rl}
\left(-\mathbf{E}+\mathbf{G}\left(\tilde{\mathscr{I}}_{0, k}\right)\right) \delta \vec{w}_{k} & =-\mathbf{E} \vec{w}_{k}-\vec{f}_{k}, \\
\vec{w}_{k+1} & =\vec{w}_{k}+\tau_{k} \delta \vec{w}_{k},
\end{array} \quad k=1,2, \ldots\right.
$$

We use a backtracking-like line search [7] to determine the step size $\tau_{k}$. Let $J_{h}$ denote a discrete approximation of the cost $J$ then $\tau_{k}$ is computed as follows

$$
\left\{\begin{aligned}
\tau_{k} & =\min _{\tau \in \mathscr{T}} J_{h}\left(\vec{w}_{k}+\tau \delta \vec{w}_{k}\right), \\
\mathscr{T} & :=\left\{\tau=\frac{2 \ell}{L} \mid \ell=1, \ldots, L\right\},
\end{aligned}\right.
$$

where $\vec{w}_{k}$ denotes the current iterate and $\delta \vec{w}_{k}$ the currently computed Newton direction. This method has proven to provide good performance and less total computational cost than standard ArmijoGoldstein or Wolfe-Powell techniques [20] (especially since no expensive evaluations of $\mathbf{G}\left(\tilde{\mathscr{I}}_{0, k}\right)$ are needed).

A combination of residual error and smallest change of iterates is used as stopping criterion in (27). The right hand side of (26) corresponds to the discretized Euler-Lagrange equations (18) of the original minimization problem (6). This motivates stopping the iteration (27) if the (relative) residual error

$r_{b}:=\left|-E \vec{w}_{k}-\vec{f}_{k}\right| /\left|-E \vec{w}_{1}-\vec{f}_{1}\right|$ is smaller than some tolerance. Additionally we compute the relative change of iterates $r_{e}:=\left|\vec{w}_{k}-\vec{w}_{k-1}\right| /\left|\vec{w}_{k}\right|$ (where $r_{e}:=0$ if $\left|\vec{w}_{k}\right|=0$ ) and combine these two notions in a stopping criterion. 
As mentioned above the choice of $\varepsilon$ may have negative effects on the outcome of the registration. Too small values of $\varepsilon$ may produce a trivial solution. Very large values of $\varepsilon$ can lead to a loss of potentially important information on the original edges $\Gamma_{i}$. Thus we developed a solution strategy of the registration problem (6) which is robust against the choice of $\varepsilon$. To compensate for the approximation error introduced by replacing the binary edges $I_{i}$ with the fuzzy edge maps $\mathscr{I}_{i}$ we augmented Newton's method (27) with an outer loop that reduces blurring in $\mathscr{I}_{i}$ and thus iteratively refines the computed deformations. We start by computing a "rough" initial guess $\vec{w}^{\star}$ by using a low number of maximal iterations $k_{\max }$ in (27). Then we compute the element-wise-squared of $\tilde{\mathscr{I}}_{i}^{\varepsilon}$. According to definition (3) the fuzzy edge maps have intensity one at all points where the raw edges $I_{i}$ are nonzero, i.e., on $\Gamma_{i}$, and have an intensity less than one everywhere else. Thus by computing the element-wise squared of $\tilde{\mathscr{I}}_{i}^{\varepsilon}$ edge-set-pixels still have intensity one whereas their blurred surroundings end up having a lower intensity. Hence the original edge sets $\Gamma_{i}$ are accentuated. Then we restart Newton's method using the images $\tilde{\mathscr{I}}_{i}^{\varepsilon}\left(\mathbf{x}_{j}\right)^{2}$, the initial guess $\vec{w}_{1}=\vec{w}^{\star}$ and increase $k_{\text {max }}$. Repeating this procedure sufficiently many times yields fuzzy edges that are very close approximations to the raw edges. Details are given in Algorithm 1.

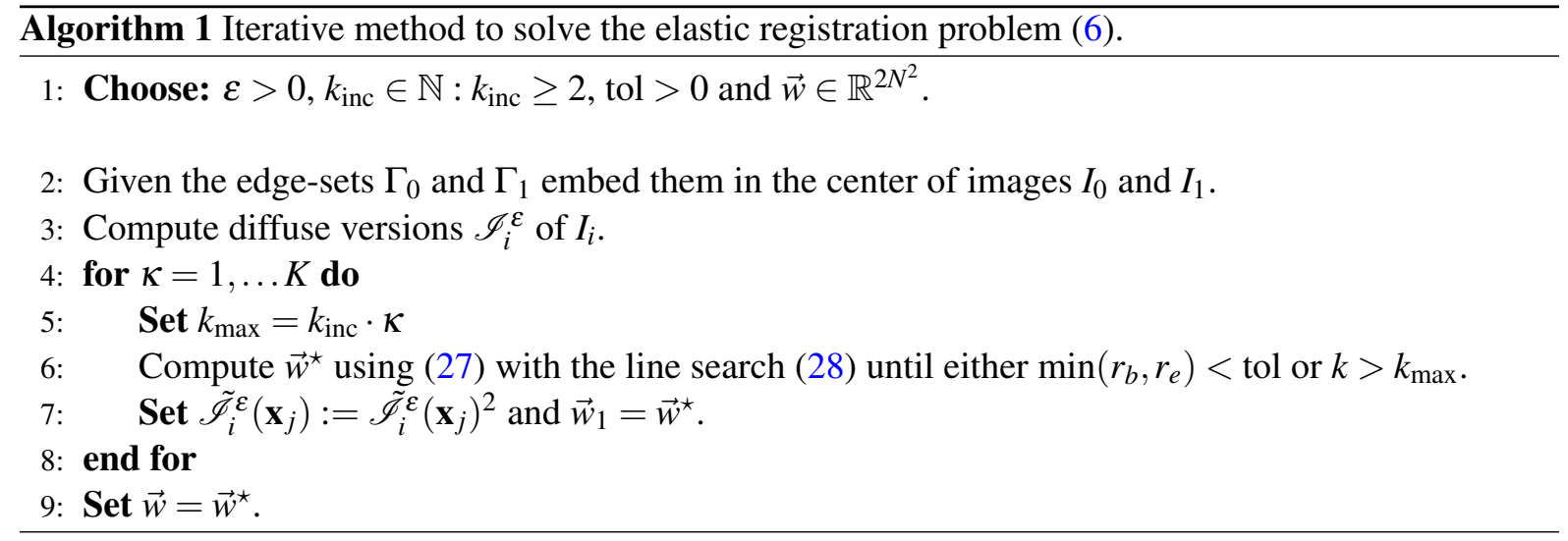

\section{Computational Results}

All computations were carried out on a 64bit Linux workstation in MATLAB ${ }^{\mathrm{TM}} 2009 \mathrm{~b}$ running on a Dell Optiplex 745 equipped with 8 GB of RAM.

The objective of this work was to map a literature-based PS available for the San Diego rabbit ventricles [28, 29] onto the endocardial surfaces of an anatomically highly realistic heart model [4] (referred to as "Oxford-heart"). Both models are shown in Fig. 2. Following an elastic registration approach, we were able to determine the transformation field between the complex endocardial surfaces of both hearts. Since the PS was embedded in the surfaces of the template heart model, it was self-evident to apply this transformation to the given PS to map the network-like structure onto the endocardial surfaces of the Oxford-heart. In this way topological features as well as the relative position of single network nodes on the ventricular surfaces could be preserved.

Following steps were pursued to apply the proposed solution strategy presented above: the 3D models were sliced to obtain sets of edges in 2D. Each slice was then registered consecutively employing Algorithm 1. The deformation fields computed for each slice were used to map the literaturebased Purkinje fiber network onto the endocardium of the Oxford-heart.

Prior to slicing the hearts we applied a (linear) translation to the models to get a maximal spatial overlap. Furthermore, to ensure a clear discrimination between left and right cavity we separated the $3 \mathrm{D}$ heart models accordingly. Once left and right cavities were separated, the $z$-axis was the obvious choice for the normal of the cutting plane.

In this fashion we generated a stack of binary images onto which Algorithm 1 was applied. After centering the edges within the binary images we employed our "region-growing" method to obtain blurred approximations $\mathscr{I}_{i}^{\varepsilon}$. Note that we approximated the distance function $d_{\Gamma_{i}}$ appearing in the definition (3) of $\mathscr{I}_{i}^{\varepsilon}$ by a marching procedure using successive discrete convolutions with a $3 \times 3$ kernel of ones. A representative result of this region-growing is depicted in Fig. 3 (c). Thus we generated two image stacks: the blurred San Diego rabbit ventricle slices $\left\{\mathscr{I}_{0}^{\varepsilon, m}\right\}_{m=1}^{M}$ (the template image stack) and the blurred Oxford-heart-cuts $\left\{\mathscr{I}_{1}^{\varepsilon, m}\right\}_{m=1}^{M}$ (the reference image stack). The next 


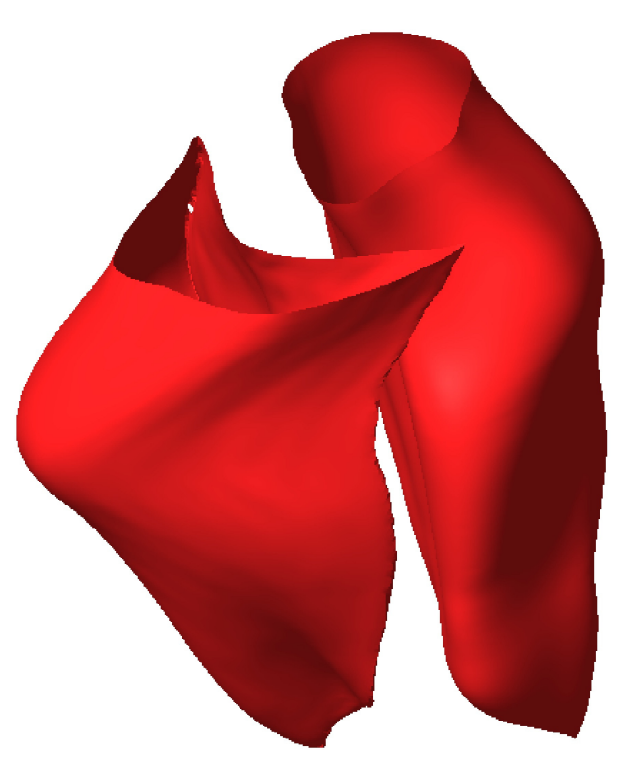

(a)

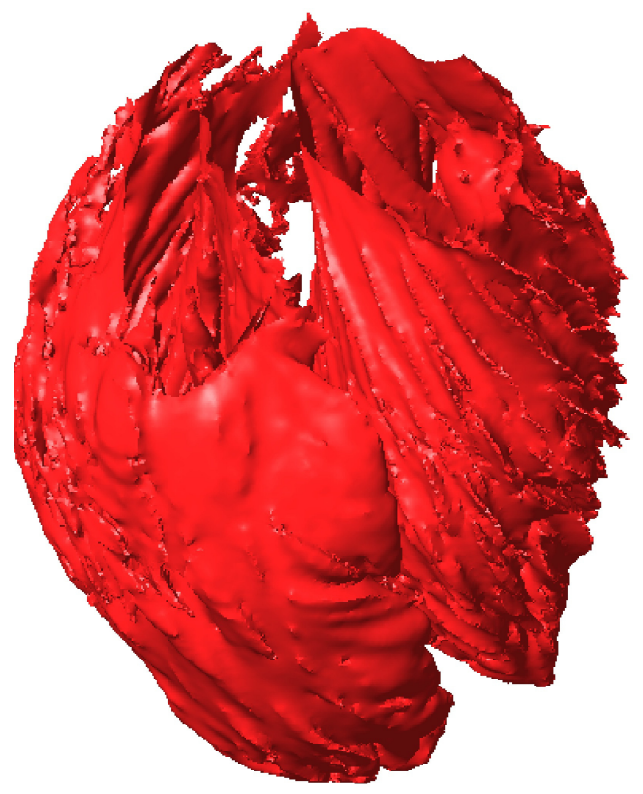

(b)

Figure 2: 3D surface representations of the ventricular cavities: (a) The San Diego rabbit ventricles [28] with a spatial discretization $\Delta x$ of $\sim 250 \mu \mathrm{m}$ serves as template geometry. (b) An anatomically highly realistic model of the ventricular cavities [4] with a $\Delta x$ of $\sim 100 \mu \mathrm{m}$ provides the target geometry during the mapping process.

Table 1: The used parameter values.

\begin{tabular}{c|c|c|c|c|c} 
Parameter & Value & Meaning \\
\hline$\lambda$ & $1 \mathrm{e}-2$ & see (5) \\
\hline$\mu$ & $1 \mathrm{e}-2$ & see (5) \\
\hline$L$ & 10 & see (28)
\end{tabular}$\quad$\begin{tabular}{ccc} 
Parameter & Value & Meaning \\
\hline tol & $1 \mathrm{e}-3$ & see Algorithm 1 \\
\hline$K$ & 4 & see Algorithm 1 \\
\hline$k_{\text {inc }}$ & 5 & see Algorithm 1
\end{tabular}$\quad \quad \begin{gathered} \\
\end{gathered}$

step was to compute elastic deformations $\mathbf{w}^{m}$ such that $\mathscr{I}_{0}^{\varepsilon, m}\left(\mathbf{x}+\mathbf{w}^{m}\right) \approx \mathscr{I}_{1}^{\varepsilon, m}(\mathbf{x})$ for $m=1, \ldots, M$ which was done by using a MATLAB-implementation of the augmented Newton method depicted in Algorithm 1. The computed deformations $\mathbf{w}^{m}$ were then applied to the raw binary images $I_{0}^{m}$ to register the edges $\Gamma_{0}^{m}$ to $\Gamma_{1}^{m}$. Fig. 4 sketches the procedure. Fig. 5 shows the $3 \mathrm{D}$ reconstruction of the registered San Diego rabbit ventricle slices in comparison to the 3D reference, i.e. the Oxford heart, for both left and right cavities. Finally we used the computed deformation fields to map the literature-based Purkinje fiber network (given as Cartesian coordinates of points representing the spatial locations of the nodes of a 3D graph) from the San Diego heart model onto the Oxford heart. The result is depicted in Fig. 6. A list of all parameters used and their values is given in Table 1.

\section{Discussion and Conclusion}

The application addressed here required the registered PS to be a subset of the Oxford heart, i.e. the computed deformations had to be sufficiently accurate to avoid network nodes being projected off the endocardial walls. This requirement together with the very complex geometries of the considered heart models made the construction of affine linear mappings to project the network infeasible. The computed elastic deformations guarantee that despite even large differences in the endocardial geometries of both models the artificial Purkinje fiber network is mapped sufficiently close to the Oxford endocardium. The disadvantage of using a linear elastic regularization in this context is that 


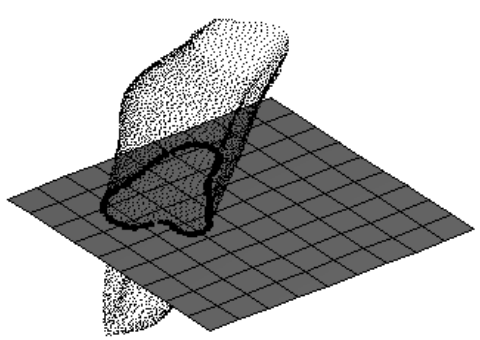

(a)

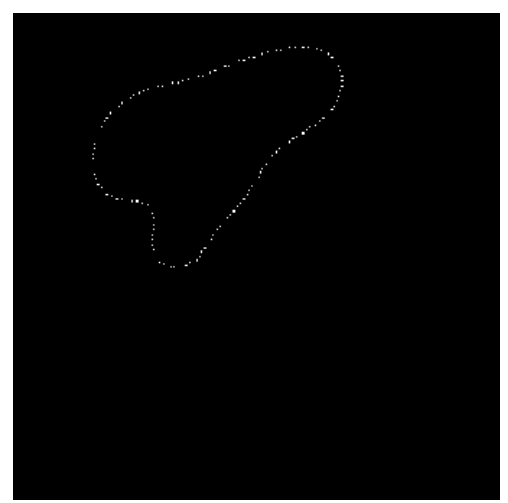

(b)

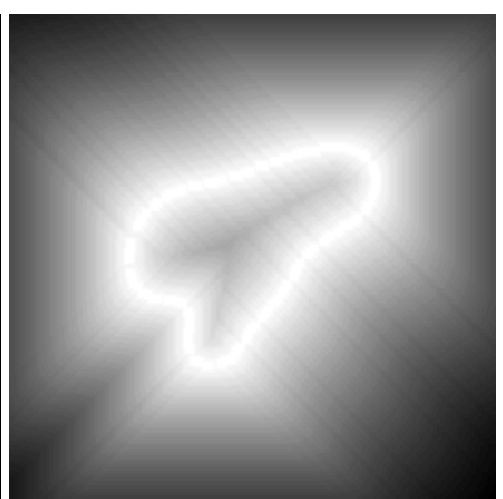

(c)

Figure 3: Illustration of the dissection of the 3D heart models. (a) shows the surface point cloud (left cavity) of the San Diego rabbit heart with a cutting plane present. First the model is cut in horizontal direction (a) to generate a 2D edge set (b). We center the image and apply our region-growing algorithm (c).

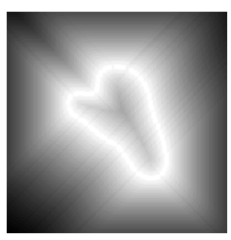

(a) $\mathscr{I}_{0}^{\varepsilon}$

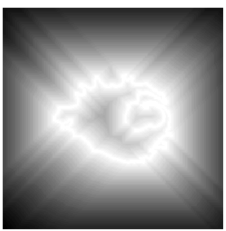

(e) $\mathscr{I}_{1}^{\varepsilon}$

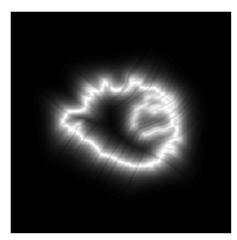

(b) $\mathscr{I}_{0}^{\varepsilon}(\mathbf{x}+\mathbf{w})^{2^{K}}$

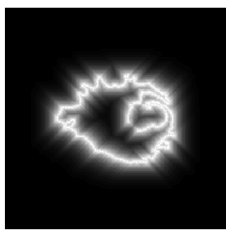

(f) $\mathscr{I}_{1}^{\varepsilon}(\mathbf{x})^{2^{K}}$

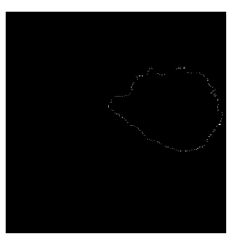

(c) $I_{0}(\mathbf{x}+\mathbf{w})$

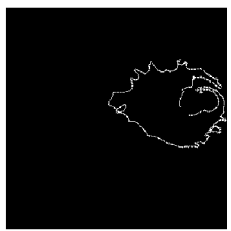

(g) $I_{1}(\mathbf{x})$

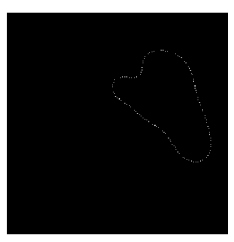

(d) $I_{0}(\mathbf{x})$

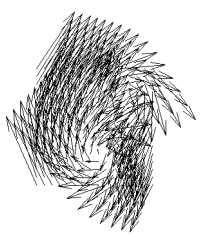

(h) $\mathbf{w}(\mathbf{x})$

Figure 4: The different stages of Algorithm 1 for the template image $I_{0}$ (top row) and the reference image $I_{1}$ (bottom row). Panels (a) and (e) show the centered and region grown versions of the images. Panel (b) presents the resulting image $\mathscr{I}_{0}^{\varepsilon}(\mathbf{x}+\mathbf{w})^{2^{K}}$ after completion of Algorithm 1 versus the final reference $\mathscr{I}_{1}^{\varepsilon 2^{K}}(\mathbf{x})$ (f). Panel (c) depicts the original binary image $I_{0}$ after application of the computed transformation $\mathbf{w}$ versus the original binary target $I_{1}(\mathrm{~g})$. The original binary template is given in panel (d). Panel (h) shows the computed deformation field $\mathbf{w}(\mathbf{x})$

the computed deformations are highly nonlinear (compare for instance Fig. 4 (h)). In a linear elastic registration scheme the principal assumption is, however, that the reference image and the template image are two observations of the same elastic body before and after a deformation. The linear elastic potential is thus a model for the displacement of the body that is only valid for sufficiently small deformations (compare [18]). This assumption is clearly violated in our context. However, here the elastic registration framework is embedded into our vanishing diffusion strategy; the most pronounced non linearities in the deformation fields are due to the first (outer) loop of Algorithm 1. But in the first loop we only use a very low number of maximal iterations $k_{\max }$ in (26). This together with a suitable choice of the Navier-Lamé constants allowed us to circumvent the restrictions of the linear elastic penalizer.

Though our results have been positively evaluated by experts, the lack of experimental observations makes a rigorous validation difficult. While our $2 \mathrm{D}$ approach depends strongly upon the 


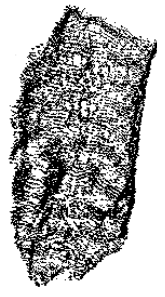

(a)

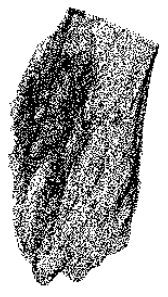

(b)

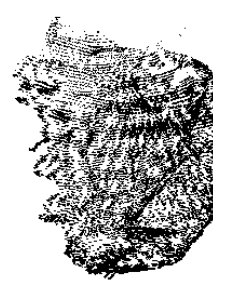

(c)

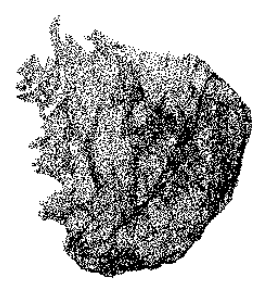

(d)

Figure 5: 3D Reconstruction of the registered San Diego rabbit ventricle slices for the left (a) and right (c) cavity and the left (b) and right (d) cavity of the Oxford heart reference geometry, respectively.

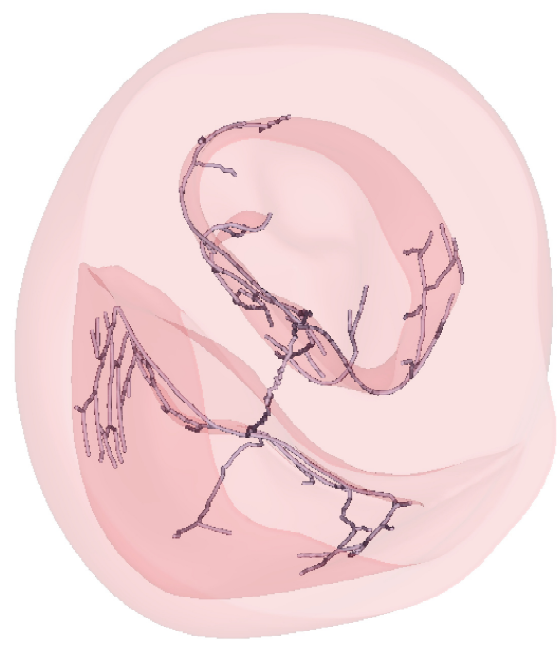

(a)

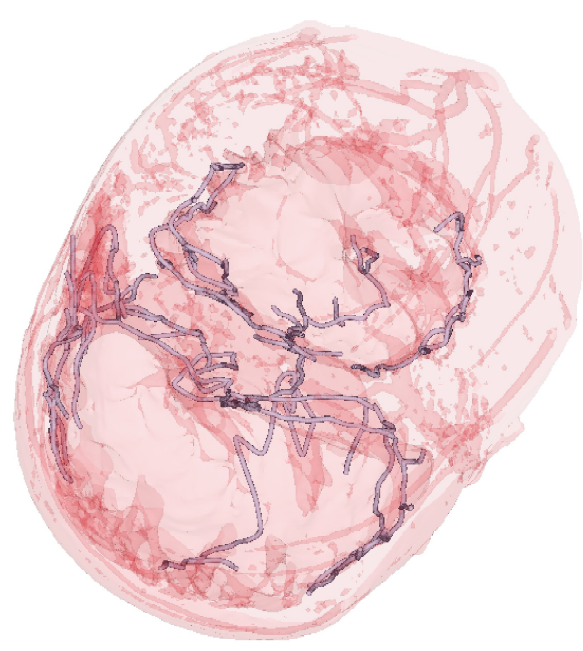

(c)

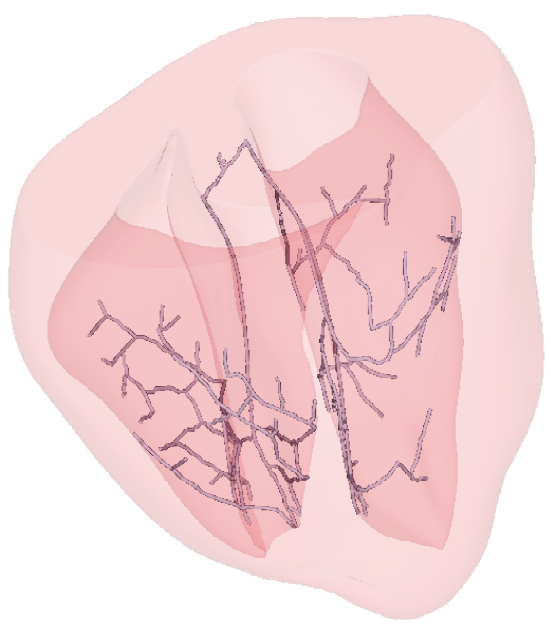

(b)

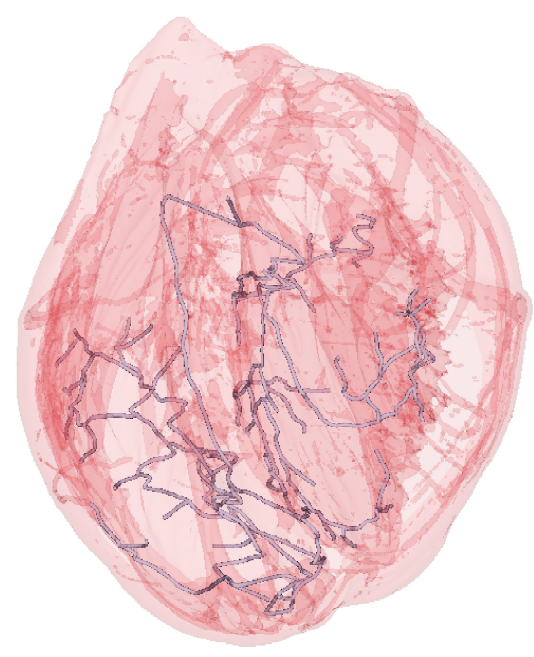

(d)

Figure 6: The artificial Purkinje fiber network in the San Diego rabbit ventricle model $(a, b)$ and the registered network in the Oxford heart (c,d).

pre-registrations performed in $3 \mathrm{D}$ and in $2 \mathrm{D}$, this dependence might be relaxed by a full $3 \mathrm{D}$ registration at considerably higher cost. Our simulations confirmed that separating the cavities of the 
heart models is crucial. By cutting the hearts in vertical direction one obtains slices containing edges from both the left and right cavities. This fact seriously impairs the outcome of the registration: the computed deformation fields may "pull" edges corresponding to the left cavity to an edge arising from a cut through the right cavity and vice versa. Hence we separated the 3D hearts into left and right cavities.

Despite the application presented here our method proved to be a highly efficient and reliable technique to register 2D edges. Forthcoming work even shows that our vanishing diffusion strategy is a really promising method in the very general context of edge set registration problems. In contrast to methods employing the Hausdorff-distance our approach allows us to use the much simpler SSID-distance which is computationally very cheap. Due to the plain structure of the associated cost functional the derivation of necessary optimality conditions by means of variational calculus is straight forward. The use of variational derivatives further enables us to employ fast and theoretically well-founded optimization routines such as Newton's method. Furthermore, the driving force of the registration can be quickly evaluated and is easy to interpret. In relation to other works employing blurring strategies in registration problems we want to emphasize that our novel vanishing diffusion strategy described in Algorithm 1 still proves to be very robust in practical use.

\section{References}

[1] L. Ambrosio and V.M. Tortorelli. Approximation of functionals depending on jumps by elliptic functionals via $\gamma$-convergence. Communications on Pure and Applied Mathematics, 43:9991036, 1990.

[2] A. Ansari, S. Y. Ho, and R. H. Anderson. Distribution of the purkinje fibres in the sheep heart. Anat Rec, 254(1):92-97, Jan 1999.

[3] Gilles Aubert and Pierre Kornprobst. Mathematical Problems In Image Processing, volume 147 of Applied Mathematical Sciences. Springer, 2nd edition, 2006.

[4] M. J. Bishop, G. Plank, R. A. Burton, J. E. Schneider, D. J. Gavaghan, V. Grau, and P. Kohl. Development of an anatomically detailed MRI-derived rabbit ventricular model and assessment of its impact on simulations of electrophysiological function. Am J Physiol Heart Circ Physiol, 298(2):H699-718, 2010.

[5] P.G. Ciarlet. The Finite Element Method for Elliptic Problems. North-Holland, Amsterdam, 1978.

[6] E. De Giorgi. Convergence problems for functionals and operators. In Proceedings of the International Meeting on Recent methods in non-linear analysis, pages 131-188, Rome 1978, 1979.

[7] J.E. Dennis and R.B. Schnabel. Numerical Methods for Unconstrained Optimization and Nonlinear Equations. Prentice-Hall, Englewood Cliffs, NJ,, 1983.

[8] M. Droske and W. Ring. A mumford-shah level-set approach for geometric image registration. SIAM Journal of Applied Mathematics, 66(6):2127-2148, 2006.

[9] J.M. Fitzpatrick, D.L.G. Hill, and C.R. Maurer. Image Registration, Medical Image Processing, volume 2, chapter 8 of the Handbook of Medical Imaging. SPIE Press, July 2000.

[10] Matthias Fuchs, Bert Jüttler, Otmar Scherzer, and Huaiping Yang. Shape metrics based on elastic deformations. J. Math. Imaging Vis., 35(1):86-102, 2009.

[11] Stefan Fürtinger, Stephen L. Keeling, Gernot Plank, and Anton J Prassl. Registration of edge sets for mapping a purkinje fiber network onto an endocardium. Technical report, Institute for Mathematics and Scientific Computing, Karl-Franzens University, Graz, Austria, 2010.

[12] Stefan Fürtinger, Stephen L. Keeling, Gernot Plank, and Anton J Prassl. Elastic registration of edge sets by means of diffuse surfaces - with an application to embedding purkinje fiber networks. In Leonid Mestetskiy and Jose Braz, editors, VISAPP, pages 12-21. SciTePress, 2011.

[13] William Hill and Richard A. Baldock. The constrained distance transform: Interactive atlas registration with large deformations through constrained distances. In DEFORM'06 - Workshop on Image Registration in Deformable Environments, 2006.

[14] R. P. Holland and H. Brooks. The qrs complex during myocardial ischemia. an experimental analysis in the porcine heart. J Clin Invest, 57(3):541-550, Mar 1976. 
[15] D. J. Huelsing, K. W. Spitzer, J. M. Cordeiro, and A. E. Pollard. Conduction between isolated rabbit purkinje and ventricular myocytes coupled by a variable resistance. Am J Physiol, 274(4 Pt 2):H1163-H1173, Apr 1998.

[16] S.L. Keeling and W. Ring. Medical image registration and interpolation by optical flow with maximal rigidity. Journal of Mathematical Imaging and Vision, 23(1):47-65, 2005.

[17] Christian Knauer, Klaus Kriegel, and Fabian Stehn. Minimizing the weighted directed hausdorff distance between colored point sets under translations and rigid motions. In $F A W$ '09: Proceedings of the 3d International Workshop on Frontiers in Algorithmics, pages 108-119, Berlin, Heidelberg, 2009. Springer-Verlag.

[18] Jan Modersitzki. Numerical Methods for Image Registration. Oxford Science Publications, 2004.

[19] D. Mumford and J. Shah. Optimal approximations by piecewise smooth functions and associated variational problems. Communications on Pure and Applied Mathematics, 42(5):577-685, 1989.

[20] J. Nocedal and S.J. Wright. Numerical Optimization. Springer, August 2000.

[21] J. M. Ortega. The newton-kantorovich theorem. The American Mathematical Monthly, 75(6):658-660, Jun-Jul 1968.

[22] Nikos Paragios and Mikael Rousson Visvanathan Ramesh. Matching distance functions: A shape-to-area variational approach for global-to-local registration. In Proceedings of the 7th European Conference on Computer Vision-Part II, 2002.

[23] Wladimir Peckar, Christoph Schnörr, Karl Rohr, and H. Siegfried Stiehl. Parameter-free elastic deformation approach for $2 \mathrm{~d}$ and $3 \mathrm{~d}$ registration using prescribed displacements. J. Math. Imaging Vis., 10(2):143-162, 1999.

[24] G. Plank, R. A. Burton, P. Hales, M. Bishop, T. Mansoori, M. O. Bernabeu, A. Garny, A. J. Prassl, C. Bollensdorff, F. Mason, F. Mahmood, B. Rodriguez, V. Grau, J. E. Schneider, D. Gavaghan, and P. Kohl. Generation of histo-anatomically representative models of the individual heart: tools and application. Philos Transact A Math Phys Eng Sci, 367(1896):2257-92, 2009.

[25] A. J. Prassl, F. Kickinger, H. Ahammer, V. Grau, J. E. Schneider, E. Hofer, E. J. Vigmond, N. A. Trayanova, and G. Plank. Automatically generated, anatomically accurate meshes for cardiac electrophysiology problems. IEEE Trans Biomed Eng, 56(5):1318-30, 2009.

[26] J. Toriwaki and H. Yoshida. Fundamentals of Three-dimensional Digital Image Processing. Springer, 2009.

[27] J. Tranum-Jensen, A. A. Wilde, J. T. Vermeulen, and M. J. Janse. Morphology of electrophysiologically identified junctions between purkinje fibers and ventricular muscle in rabbit and pig hearts. Circ Res, 69(2):429-437, Aug 1991.

[28] F.J. Vetter and A.D. McCulloch. Three-dimensional analysis of regional cardiac function: a model of rabbit ventricular anatomy. Prog Biophys Mol Biol, 69(2-3):157-83, 1998.

[29] Edward J Vigmond and Clyde Clements. Construction of a computer model to investigate sawtooth effects in the purkinje system. IEEE Trans Biomed Eng, 54(3):389-399, Mar 2007.

[30] Siwei Yang, D. Kohler, K. Teller, T. Cremer, P. Le Baccon, E. Heard, R. Eils, and K. Rohr. Nonrigid registration of 3-d multichannel microscopy images of cell nuclei. IEEE Transactions on Image Processing, 17:493-499, April 2008. 